This copy is for your personal, non-commercial use only.

If you wish to distribute this article to others, you can order high-quality copies for your colleagues, clients, or customers by clicking here.

Permission to republish or repurpose articles or portions of articles can be obtained by following the guidelines here.

The following resources related to this article are available online at www.sciencemag.org (this information is current as of May 31, 2012 ):

Updated information and services, including high-resolution figures, can be found in the online version of this article at:

http://www.sciencemag.org/content/336/6085/1182.full.html

Supporting Online Material can be found at:

http://www.sciencemag.org/content/suppl/2012/05/30/336.6085.1182.DC1.html http://www.sciencemag.org/content/suppl/2012/05/30/336.6085.1182.DC2.html

A list of selected additional articles on the Science Web sites related to this article can be found at:

http://www.sciencemag.org/content/336/6085/1182.full.html\#related

This article cites 32 articles, 7 of which can be accessed free:

http://www.sciencemag.org/content/336/6085/1182.full.html\#ref-list-1

This article appears in the following subject collections:

Neuroscience

http://www.sciencemag.org/cgi/collection/neuroscience 


\section{Restoring Voluntary Control of Locomotion after Paralyzing Spinal Cord Injury}

Rubia van den Brand, ${ }^{1,2 *}$ Janine Heutschi, ${ }^{1,2 *}$ Quentin Barraud, ${ }^{1,2}$ Jack DiGiovanna, ${ }^{3,4}$ Kay Bartholdi, ${ }^{1,2}$ Michèle Huerlimann, ${ }^{1}$ Lucia Friedli, ${ }^{1,2}$ Isabel Vollenweider, ${ }^{1,2}$ Eduardo Martin Moraud, ${ }^{3,4}$ Simone Duis, ${ }^{1,2}$ Nadia Dominici, ${ }^{1,2}$ Silvestro Micera, ${ }^{3,4}$ Pavel Musienko, ${ }^{1,2}$ Grégoire Courtine ${ }^{1,2} \dagger$

Half of human spinal cord injuries lead to chronic paralysis. Here, we introduce an electrochemical neuroprosthesis and a robotic postural interface designed to encourage supraspinally mediated movements in rats with paralyzing lesions. Despite the interruption of direct supraspinal pathways, the cortex regained the capacity to transform contextual information into task-specific commands to execute refined locomotion. This recovery relied on the extensive remodeling of cortical projections, including the formation of brainstem and intraspinal relays that restored qualitative control over electrochemically enabled lumbosacral circuitries. Automated treadmill-restricted training, which did not engage cortical neurons, failed to promote translesional plasticity and recovery. By encouraging active participation under functional states, our training paradigm triggered a cortex-dependent recovery that may improve function after similar injuries in humans. an intervening gap of intact tissue. The lesion, however, led to a complete loss of hindlimb function, with no sign of recovery over 2 months post injury (fig. S1D). Likewise, humans with clinically complete SCI frequently show maintenance of connections through the lesion (7). Thus, this experimental lesion reproduces key anatomical and functional features of human SCIs, while providing well-controlled conditions to investigate the mechanisms underlying recovery (8).

To transform lumbosacral circuits from dormant to highly functional states (9), we applied tonic $(40 \mathrm{~Hz})$ epidural electrical stimulation over L2 and S1 spinal segments (6), and systemically administered a tailored cocktail of serotonin receptor agonists $\left(5 \mathrm{HT}_{1 \mathrm{~A} / 7}\right.$ and $\left.5 \mathrm{HT}_{2 \mathrm{~A} / \mathrm{C}}\right)$ and dopamine $\left(\mathrm{D}_{1}\right)$ receptor agonists $(10)$. By increasing the general level of spinal excitability, this electrochemical spinal neuroprosthesis enables sensory information to become a source of control for stepping $(6,9)$. This intervention promoted coordinated, although involuntary, bipedal stepping on a treadmill as early as 7 days post injury (Fig. 1C).

These stepping movements are elicited by the moving treadmill belt (6), which suggests that the

${ }^{1}$ Neurology Department, University of Zurich, $\mathrm{CH}-8008$ Zurich, Switzerland. ${ }^{2}$ Center for Neuroprosthetics and Brain Mind Institute, School of Life Sciences, Swiss Federal Institute of Technology (EPFL), CH-1015 Lausanne, Switzerland. ${ }^{3}$ Center for Neuroprosthetics, School of Engineering, Swiss Federal Institute of Technology (EPFL), CH-1015 Lausanne, Switzerland. ${ }^{4}$ Automatic Control Laboratory, Swiss Federal Institute of Technology (ETH), CH-8092 Zurich, Switzerland.

*These authors contributed equally to this work.

†To whom correspondence should be addressed. E-mail: gregoire.courtine@epfl.ch

\footnotetext{
A ctivity-based interventions exploiting proprioceptive information to enhance spinal motor output during training $(1-3)$ promote plastic changes capable of restoring locomotion after severe though incomplete spinal cord injury (SCI) $(3,4)$. A recent case study suggests that, in combination with epidural electrical stimulation of lumbosacral segments, activitybased rehabilitation may also restore supraspinally mediated movements after motor complete paraplegia (5). We aimed to design a multisystem neuroprosthetic training program that took full
}

advantage of this concept. We hypothesized that, after the complete interruption of direct supraspinal input, a robotic postural interface encouraging the brain to actively use the paralyzed hindlimbs during electrochemically enabled motor states (6) would reestablish supraspinal control of locomotion by promoting extensive and ubiquitous remodeling of spared neuronal circuitries.

Adult rats received a left lateral over-hemisection at thoracic (T) vertebra T7 and a right lateral hemisection at T10. This SCI interrupts all direct supraspinal pathways (fig. S1, A to C), but leaves
Fig. 1. Multisystem neuroprosthetic training restores voluntary locomotion after paralyzing $\mathrm{SCl}$. (A) Left hindlimb kinematics, hindlimb end-point trajectory and velocity vector, vertical ground reaction forces (vGRF), as well as electromyographic (EMG) activity of medial gastrocnemius (MG) and TA muscles during bipedal locomotion in an intact rat. (B) Robotic postural interface providing vertical and lateral support, but no facilitation in the forward direction. (C) Representative left hindlimb stepping patterns recorded under the various experimental conditions 1 and 9 weeks post SCl. (D) Distance covered in $3 \mathrm{~min}$ during bipedal locomotion. $\%$, Body weight sup-
A
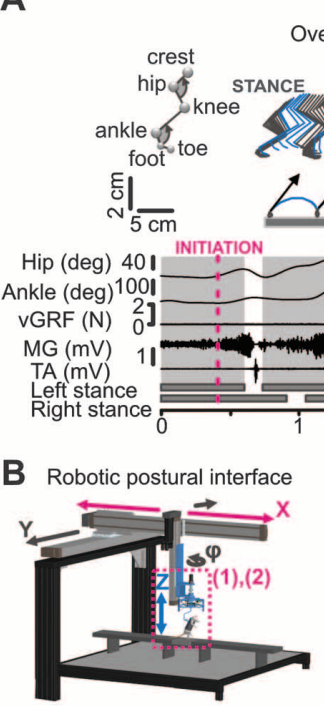

Overground $(0 \%)$
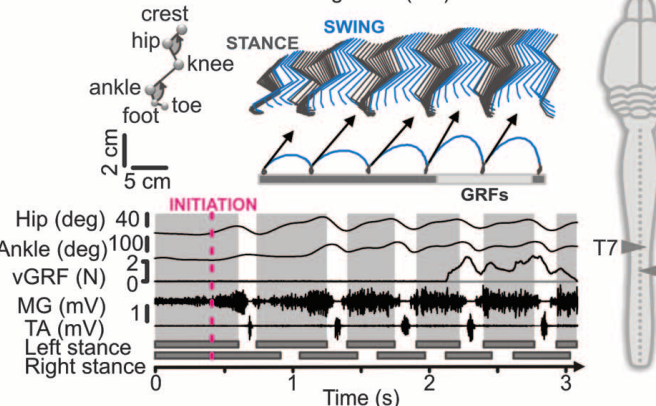

Post-injury (weeks)

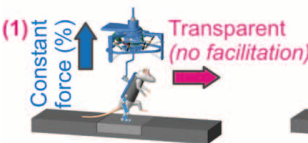

Intact

GRFs

Time (s)

D 3-minute walk test

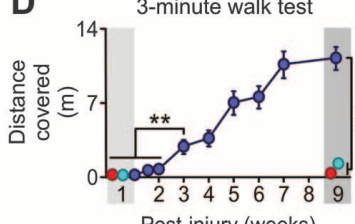

C
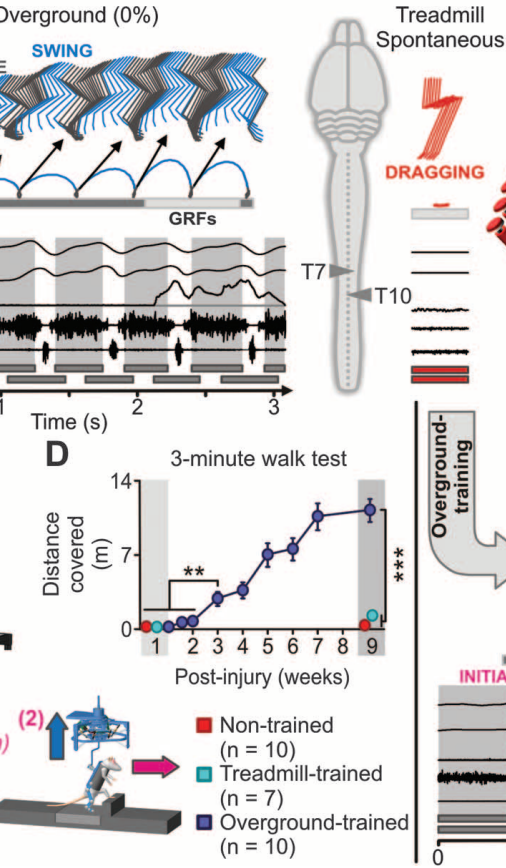

Sub-acute (1 week post-injury) Treadmill (85\%) Overground (70\%) Overground $(50 \%)$ Enabling factors Enabling factors Enabling factors

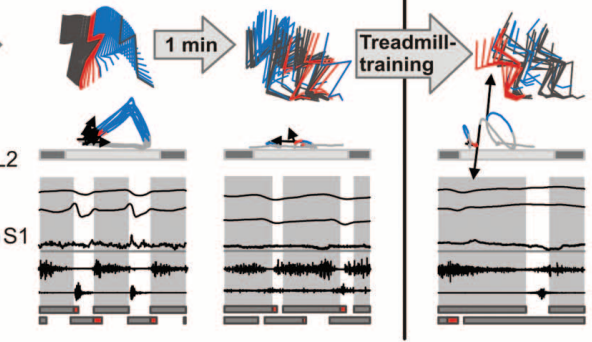

Chronic (9 weeks post-injury)

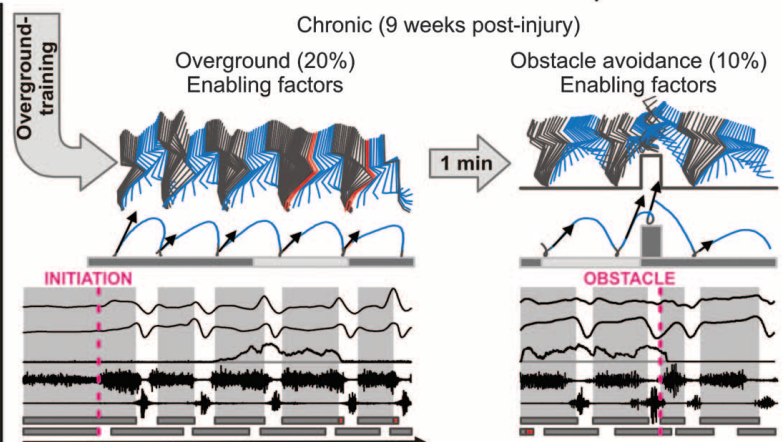


rats would not be capable of voluntarily initiating hindlimb locomotion overground. To verify the absence of supraspinal control, we applied the electrochemical neuroprosthesis and positioned the same rats bipedally in a robotic postural interface that provided adjustable vertical and lateral trunk support, but did not facilitate locomotion in any direction (Fig. 1B and fig. S2). All the rats $(n=27)$ failed to initiate hindlimb locomotion overground 7 days post injury $(P<$ 0.001) (Fig. 1C).

We then designed a multisystem neuroprosthetic training program that encompassed two objectives. First, we aimed to improve the functionality of lumbosacral circuits through treadmill-based training enabled by the electrochemical neuroprosthesis (6). Second, we sought to promote the recovery of supraspinally mediated movements; we exploited the robotic postural interface not only to enable, but also to force, the rats to actively use their paralyzed hindlimbs in order to locomote bipedally toward a target.

Rats $(n=10)$ were trained daily for $30 \mathrm{~min}$ with a combination of both paradigms, starting 7 to 8 days post injury (fig. S3). The first, effortful voluntary steps emerged after 2 to 3 weeks of training $(P<0.01)$ (Fig. 1D). As voluntary movements recovered, we gradually increased the relative duration of overground training (fig. S3B). Five to 6 weeks post injury, all the rats (fig.

S4) were capable of initiating and sustaining full weight-bearing bipedal locomotion for extended periods of time, but only during electrochemically enabled motor states (Fig. 1, C and D, fig. S1D, and movie S1). Kinematic analyses (fig. S5) revealed that overground-trained rats deployed a similar control strategy as intact animals to produce locomotion (Fig. 1, A and C, and fig. S5). To measure recovery, we adapted the clinically standardized 6-min walk test (11) to bipedally stepping rats. Overground-trained animals with a paralyzing SCI covered distances as long as $21 \mathrm{~m}$ in $3 \mathrm{~min}$ (Fig. 1D).

We next tested whether treadmill-restricted step training under electrochemically enabled states would also promote the recovery of voluntary locomotion ( $n=7$ rats). This automated step training failed to reestablish overground locomotion despite repeated testing during 4 to 8 sessions 9 weeks post injury $(P<0.001)$ (Fig. 1, C and $\mathrm{D}$, and movie S1). Moreover, treadmilltrained rats were not capable of sustaining robotically initiated locomotion overground (fig. S6).

To further enhance supraspinal contribution, we introduced stairs and obstacles; two conditions requiring voluntarily mediated gait tuning (12). After 2 to 3 additional weeks, overgroundtrained rats were capable of bipedally sprinting up stairs and avoiding obstacles (Fig. 1C, fig. S7, and movie S1). To accomplish these paradigms,

A
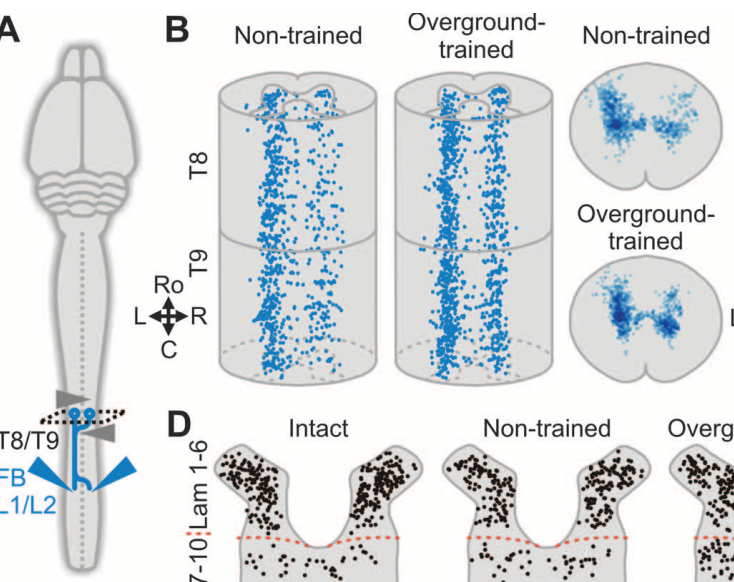

C

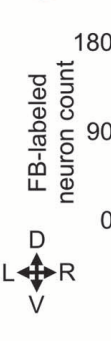

Laminae 7-10 Laft

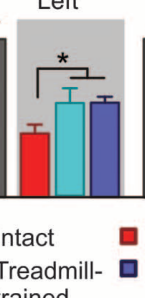
rained
E

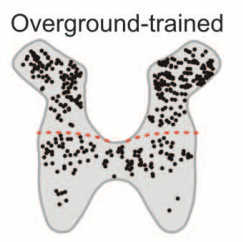

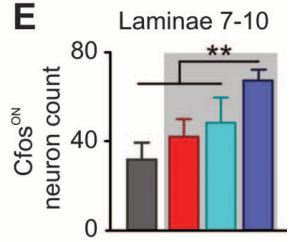

H 3-min walk test

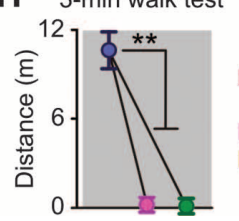

口 Overgroundtrained

- Post T6 SCl - Post NMDA
F Overground-trained

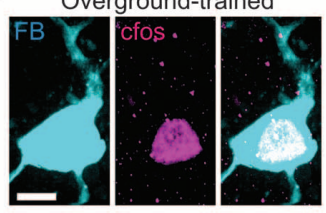

G

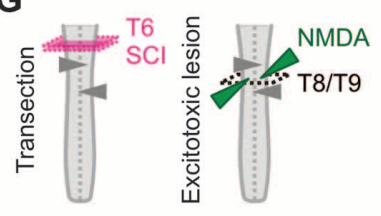

the animals displayed a range of task-specific adjustments of hindlimb movements (fig. S7).

Anatomical examinations highlighted an extensive remodeling of supraspinal and intraspinal projections in rats that regained voluntary locomotion. We first conducted retrograde tract tracing from lumbar (L) vertebrae L1/L2 locomotor centers (Fig. 2A). We found a significant increase $(P<0.05)$ (Fig. 2, B and C) in the number of labeled neurons in intermediate and ventral laminae of T8/T9 segments in both overgroundtrained and treadmill-trained rats compared with nontrained animals. Analysis of the activitydependent marker, c-fos, after continuous overground locomotion confirmed that the labeled neurons were active during walking (Fig. 2F). The number of c-fos ${ }^{\text {on }}$ nuclei in the regions rich in neurons retrogradely labeled from L1/L2 locomotor centers was larger in overgroundtrained rats compared with all the other groups $(P<0.01)$ (Fig. 2, D and E). Thoracic neurons may thus play a pivotal role in restoring voluntary locomotion $(8,13,14)$. To address this hypothesis, we ablated T8/T9 neurons by infusing the axon-sparing excitotoxin $\mathrm{N}$-methylD-aspartic acid (NMDA) (8) (Fig. 2G and fig. S8). Infusion of NMDA abolished the regained voluntary locomotion $(P<0.01)$ (Fig. $2 \mathrm{H}$ and movie S2), despite uncompromised functionality of lumbosacral circuits (fig. S8). Likewise, overground-trained rats lost voluntary control of locomotion after the complete interruption of supraspinal input to T8/T9 neurons $(P<0.01)$ (Fig. 2, $\mathrm{G}$ and $\mathrm{H}$ ).

We labeled projections from the left hindlimb motor cortex with injections of biotinylated dextran amine (BDA) (Fig. 3A). The bilateral interruption of the dorsal column at the $\mathrm{T} 7$ over-hemisection only spared a few (1 to $2 \%$ ) (15) corticospinal tract (CST) axons in the right dorsolateral funiculus (fig. S9E). Consequently, nontrained rats showed scarce CST labeling in T8/T9 segments (Fig. 3, B and C, and fig. S9E). Treadmill-restricted training did not promote significant changes in the density of thoracic CST projections (Fig. 3, B and $\mathrm{C}$, and fig. S9E). In contrast, we found a reconstitution of $45 \pm 7 \%$ of prelesion bilateral fiber density in overground-trained rats (Fig. 3, B to D). These CST axons exclusively branched from the right dorsolateral funiculus (Fig. 3D), and they profusely innervated the right and, more unexpectedly, the left gray matter of T8/T9 segments (fig. S9F) (16). We detected multiple CST fibers extending from the gray matter at the $\mathrm{T} 7$ lesion site into the right dorsolateral funiculus (Fig. 3, $\mathrm{E}$ and $\mathrm{F}$ ). These ectopic fibers, suggestive of regenerative sprouting (17), led to a near twofold increase in the CST axon density of the T8/T9 dorsolateral funiculus $(P<0.01)$ (fig. S9G). Thoracic CST fibers bypassed the T7 overhemisection through the right dorsolateral funiculus, branched into the gray matter, and recrossed the midline (Fig. 3E). These fibers developed large axonal structures with boutonlike swellings

suggestive of sprouting in terminal arbors (fig. S9F).
Fig. 2. Multisystem neuroprosthetic training promotes the formation of intraspinal detours that relay supraspinal information. (A) Diagram illustrating anatomical experiments. (B) Longitudinal and transverse views of three-dimensional (3D) reconstructions of Fast blue-labeled (FB) neurons between the lesions. L, left; R, right; Ro, rostral; C, caudal; D, dorsal; V, ventral. (C) Counts ( $n=6$ to 9 rats per group) of FB neurons in laminae 7 to 10 of T8/T9 segments after 45 min of continuous locomotion. (D) C-fos expression patterns in T8/T9 segments. (E) Counts $\left(n=5\right.$ to 7 rats per group) of $c-f_{0}{ }^{\text {ON }}$ neurons in laminae 7 to 10 after continuous locomotion. (F) Colocalization of FB and c-fos. Scale bar, $10 \mu \mathrm{m}$. (G) Overground-trained rats received a complete T6 SCI $(n=2)$ or T8/T9 NMDA microinjections $(n=3)$. (H) Distance covered in 3 min before and after the lesions. ${ }^{*} P<0.05$. ${ }^{*} P<0.01$. Error bars, SEM. 

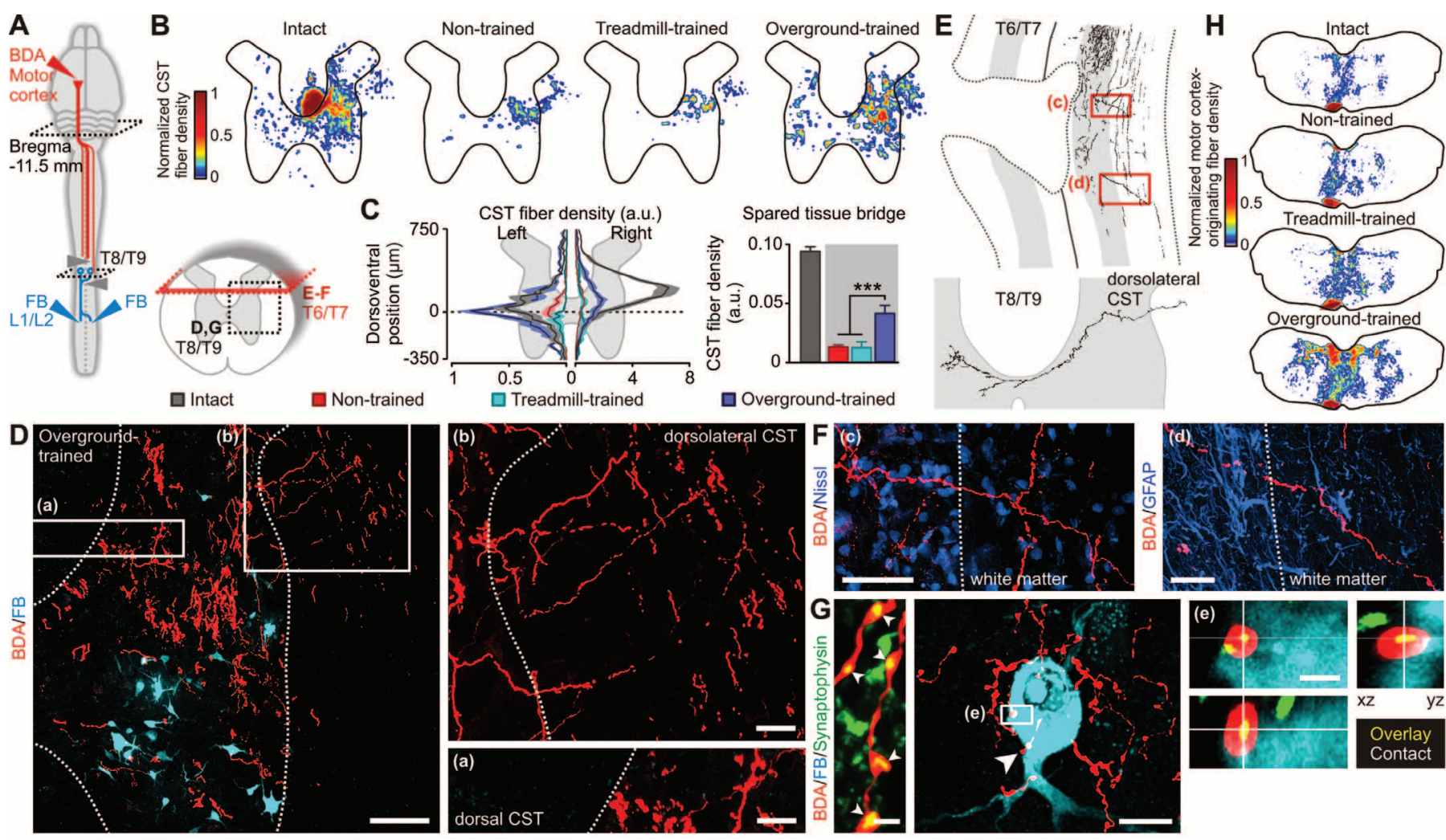

Fig. 3. Multisystem neuroprosthetic training promotes extensive remodeling of motor cortex projections. (A) Diagram illustrating anatomical experiments and analyzed regions. (B) Heat maps and (C) graphs ( $n=5$ rats per group) showing bilateral CST axon density in T8/T9 segments, a.u., arbitrary units. (D) Confocal overview of the right T8/T9 hemicord of an overground-trained rat. Scale bar, $100 \mu \mathrm{m}$; insets [(a) and (b)], $30 \mu \mathrm{m}$. (E) 3D CST fiber reconstructions

along the longitudinal (top) and transverse (bottom) plane. (F) Confocal images of insets shown in (E). Scale bar, $50 \mu \mathrm{m}$. (G) Colocalization of CST fibers with synaptophysin (arrows), and close appositions (inset and arrow) with a FB neuron. Scale bar, $2 \mu \mathrm{m}$; overview neuron, $10 \mu \mathrm{m}$. (H) Heat maps showing density of cortical projections in the brainstem (bregma $-11.5 \mathrm{~mm}$ ). $* * * P<0.001$. Error bars, SEM.
Confocal microscopy confirmed that thoracic CST fibers bore synaptic elements because they colocalized with synaptophysin (Fig. 3G). These fibers established contacts with relay neurons retrogradely labeled from L1/L2 locomotor centers (Fig. 3G).

Remodeling of motor cortex axonal projections was not restricted to the spared tissue bridge. Quantification of CST fibers at T4/T5, above the injury, revealed a significant bilateral increase of axon density in overground-trained compared with nontrained, treadmill-trained, and intact rats $(P<0.01)$ (fig. S9, A to D). We found a near fourfold increase in the density of cortical projections in various brainstem motor areas (Fig. $3 \mathrm{H}$ and fig. S10), including the left and right vestibular nuclei $(P<0.01)$, the entire reticular formation $(P<0.001)$, and parapyramidal regions $(P<0.01)$. These areas contain reticulospinal neurons and spinally projecting serotonergic neurons (fig. S10C) that both contribute to initiating and sustaining locomotion $(18,19)$. Descending 5HT fibers might thus reorganize with training. We found a nearly complete, laminaspecific restoration of T8/T9 serotonergic innervation in overground-trained rats, which contrasted with the depletion of 5HT fibers in nontrained and treadmill-trained animals $(P<0.05)$ (fig. S11).
Collectively, these analyses demonstrate that automated treadmill-restricted training failed to mediate anatomical changes in descending pathways, whereas active training under highly functional states promoted multilevel plasticity in cortex- and brainstem-derived axonal systems.

Contrary to primates, the rodent motor cortex is not essential to produce locomotion (20). Consequently, we sought to demonstrate that traininginduced remodeling of motor cortex projections did contribute to controlling voluntary locomotion. First, we implanted stimulating epidural electrodes over the left motor cortex to verify that the reorganization of neuronal pathways reestablished connectivity across the lesion. Before the SCI, applying a train of low intensity ( 0.7 to $1.5 \mathrm{~mA})$ electrical stimuli evoked large responses in the left tibialis anterior (TA) muscle (Fig. 4A). The SCI permanently abolished these responses in nontrained rats $(P<0.001)$ (Fig. 4A). In contrast, overground-trained rats regained responses below the lesion, averaging about $10 \%$ of their prelesion amplitude $(P<0.001)$ (Fig. 4B). These responses were delayed by $12 \pm 3 \mathrm{~ms}(P<0.01)$ (Fig. 4A), which suggests that a larger number of synaptic relays was necessary to convey the supraspinal volley to hindlimb motor pools. The amplitude of responses substantially increased during electrochemically enabled motor states $(P<0.01)$ (Fig. 4, A and B), which indicated enhanced transmission of the supraspinal command (14). Second, we implanted a microwire array in the vicinity of CST neurons projecting to T8/T9 segments (Fig. 4C) and recorded neuronal modulations during voluntary locomotion in overground-trained rats $(n=3)$. We found a variety of neurons $(n=$ $17 / 24$ neurons) whose modulation patterns significantly $(P<0.05)$ (Fig. 4D) correlated with gait initiation, sustained locomotion, and corrective movements (fig. S12 and movie S3). A substantial number of motor cortex neurons (36\%) exhibited a sharp increase in firing rate before any overt movement or locomotor-related muscle activity had occurred (Fig. 4E). Instead, the firing rate of motor cortex neurons significantly decreased during involuntary locomotion compared with quiet standing $(P<0.05)$ (fig. S13, A to $\mathrm{C})$. Third, we inactivated the left motor cortex with a microinjection of the $\gamma$-aminobutyric acid (GABA) agonist muscimol (Fig. 4F). Muscimol immediately suppressed voluntary hindlimb locomotion $(P<0.01)$ (Fig. 4G and movie S3), despite uncompromised functionality of lumbosacral circuits (fig. S14).

Thus far, functional restoration after SCI has been interpreted as the need to promote longdistance regeneration of severed fibers to their 
A
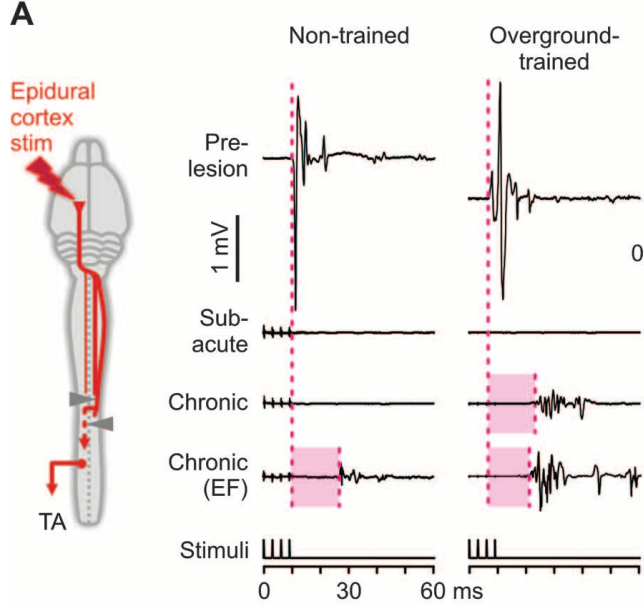

B motor responses

Non-trained Overgroundtrained EF: Enabling factors
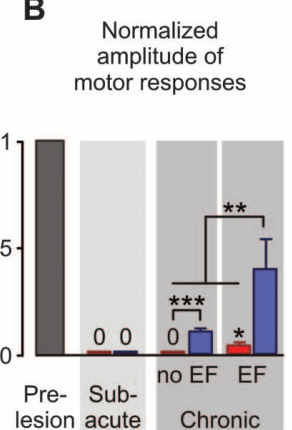

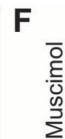
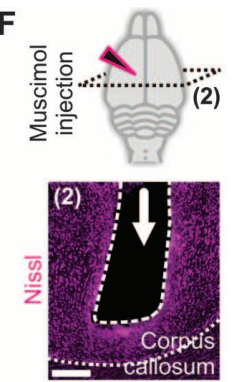

G 3-minute walk test
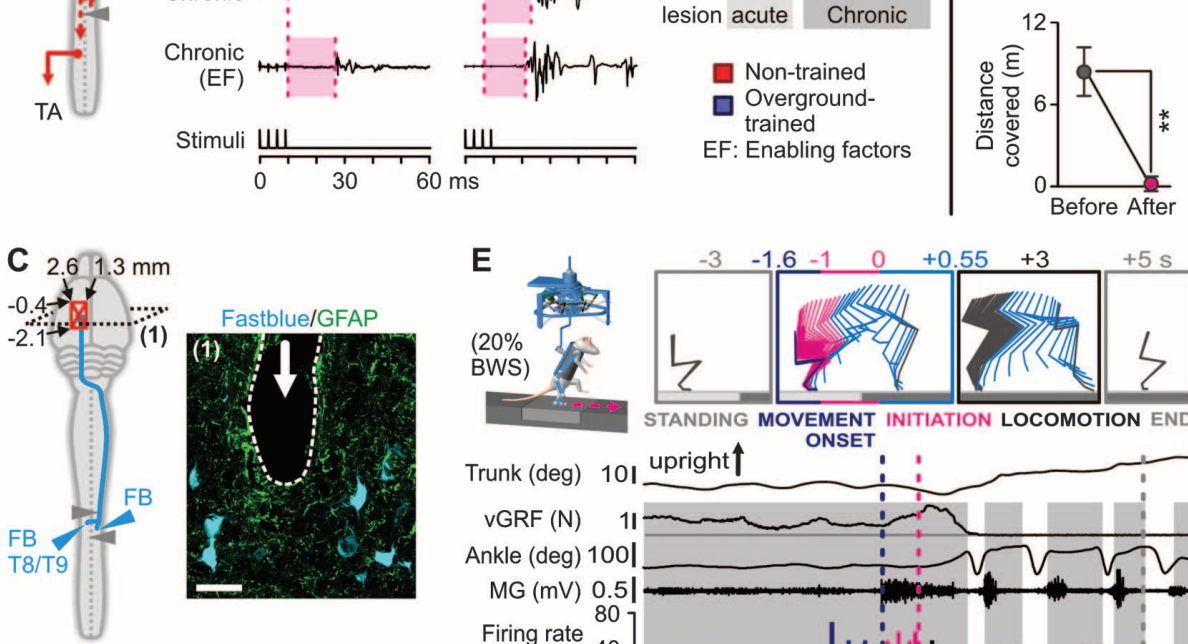

D
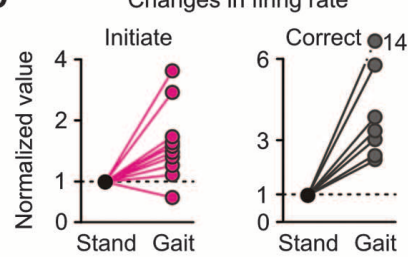

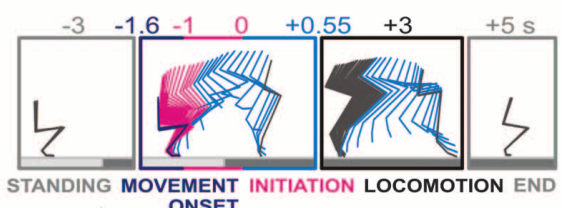
ONSET
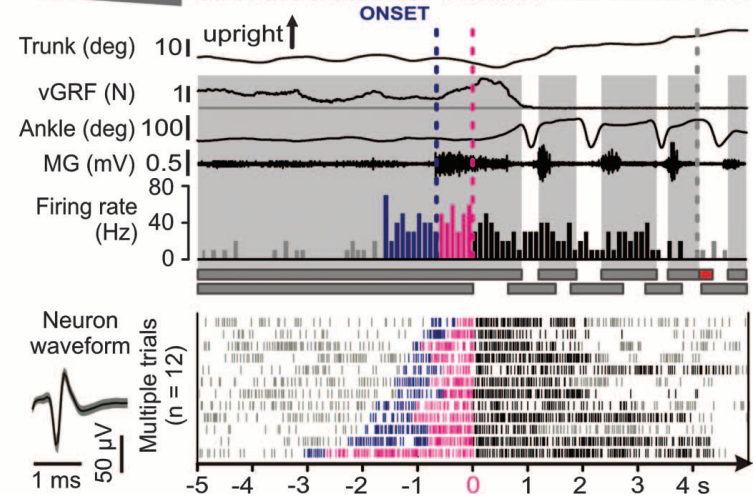

Fig. 4. Overground-trained rats regained cortical control of hindlimb locomotion. (A) Responses evoked by a train of epidural motor cortex stimulations in the left TA muscle in a nontrained and overgroundtrained rat. (B) Mean ( $n=5$ rats per group) amplitude of responses. (C) Diagram and GFAP staining to reveal glial fibrillary acidic protein (1) illustrating microwire array localization. Scale bar, $50 \mu \mathrm{m}$. (D) Change in firing rate for significantly modulated neurons during initiation and correction compared with standing. (E) Trunk vertical position, vGRFs, left ankle joint angle, EMG activity of left MG muscle, and modulation of a motor cortex neuron during one trial. Raster of the same neuron for multiple trials. Movement onset and initiation are defined as hip extension and foot clearance, respectively. The color-coding indicates the period during which firing rate significantly increased before these events. (F) Diagram and Nissl staining (2) showing catheter location for muscimol microinjection. Scale bar, $300 \mu \mathrm{m}$. (G) Distance covered in 3 min before and after muscimol injection. ${ }^{*} P<0.05$. ${ }^{* *} P<0.01$. ${ }^{* *} P<0.001$. Error bars, SEM.

original targets $(21,22)$. Undoubtedly, neuroregeneration will be essential following near-complete SCI. However, a more immediate approach might capitalize on the remarkable capacity of spared neuronal systems to reorganize through usedependent mechanisms $(3,5,23)$. Here, we established training conditions that not only enabled but also forced the brain to construct a multiplicity of de novo brainstem and intraspinal relays to regain quantitative and qualitative access to electrochemically enabled lumbosacral circuitries. There is growing evidence that active training with appropriate sensory cues is markedly superior to passive, robot-guided rehabilitation to improve stepping capacities in humans (3, 5, 23-26). Likewise, automated treadmill-restricted training, which did not engage cortical neurons, promoted sublesional plasticity, but failed to promote remodeling of descending pathways. Treadmill-trained rats did not regain supraspinally mediated locomotion. Instead, our new training paradigm encouraged active rat participation and triggered a cortexdependent, activity-based process that restored voluntary control over sophisticated locomotor movements after a SCI that led to chronic paralysis. These results confirm the capacity of intraspinal circuits to bypass lesions $(8,13)$ and expand the therapeutic potential of detour circuits to the restoration of function after paralyzing SCI. The ability of training under highly functional states to promote this extensive plasticity and recovery may lead to novel interventions capable of improving function in humans with a range of neuromotor disorders $(5,27,28)$.

References and Notes

1. H. Barbeau, S. Rossignol, Brain Res. 412, 84 (1987).

2. R. G. Lovely, R. ]. Gregor, R. R. Roy, V. R. Edgerton, Exp. Neurol. 92, 421 (1986).

3. A. Wernig, S. Müller, Paraplegia 30, 229 (1992).

4. A. Wernig, S. Müller, A. Nanassy, E. Cagol, Eur. J. Neurosci. 7, 823 (1995).

5. S. Harkema et al., Lancet 377, 1938 (2011).

6. G. Courtine et al., Nat. Neurosci. 12, 1333 (2009).

7. B. A. Kakulas, J. Spinal Cord Med. 22, 119 (1999).

8. G. Courtine et al., Nat. Med. 14, 69 (2008).

9. P. Musienko, ]. Heutschi, L. Friedli, R. V. den Brand, G. Courtine, Exp. Neurol. 235, 100 (2012).

10. P. Musienko et al., J. Neurosci. 31, 9264 (2011).

11. G. H. Guyatt et al., Can. Med. Assoc. J. 132, 919 (1985).

12. T. Drew, ]. E. Andujar, K. Lajoie, S. Yakovenko, Brain Res. Brain Res. Rev. 57, 199 (2008)

13. F. M. Bareyre et al., Nat. Neurosci. 7, 269 (2004).

14. K. C. Cowley, E. Zaporozhets, B. J. Schmidt, J. Physiol. 586, 1623 (2008).

15. C. Brösamle, M. E. Schwab, J. Comp. Neurol. 386, 293 (1997).

16. E. S. Rosenzweig et al., Nat. Neurosci. 13, 1505 (2010).

17. O. Steward, B. Zheng, M. Tessier-Lavigne, J. Comp. Neurol. 459, 1 (2003).

18. M. Hägglund, L. Borgius, K. J. Dougherty, O. Kiehn, Nat. Neurosci. 13, 246 (2010).

19. J. Liu, L. M. Jordan, J. Neurophysiol. 94, 1392 (2005).

20. G. Courtine et al., Nat. Med. 13, 561 (2007).

21. L. T. Alto et al., Nat. Neurosci. 12, 1106 (2009).

22. F. Sun et al., Nature 480, 372 (2011).

23. V. R. Edgerton et al., Brain Res. Brain Res. Rev. 57, 241 (2008)

24. L. L. Cai et al., J. Neurosci. 26, 10564 (2006).

25. A. Wernig, Arch. Phys. Med. Rehabil. 86, 2385 (2005).

26. M. Wirz et al., Arch. Phys. Med. Rehabil. 86, 672 (2005).

27. P. Musienko, R. van den Brand, O. Maerzendorfer, A. Larmagnac, G. Courtine, IEEE Trans. Biomed. Eng. 56, 2707 (2009).

28. R. Fuentes, P. Petersson, W. B. Siesser, M. G. Caron M. A. Nicolelis, Science 323, 1578 (2009).

Acknowledgments: We thank U. Keller for assistance with the robotic interface. Imaging was performed using equipment maintained by the Center for Microscopy and Image Analysis, University of Zurich. Financial support was provided by a Starting Grant from the European Research Council (ERC 261247, Walk Again); an International Paraplegic Foundation Fellowship; the Neuroscience Center Zurich; European Commission's Seventh Framework Program (CP-IP 258654, NeuWALK); and funding from the Swiss National Science Foundation (subsidy 310030_130850).

\section{Supplementary Materials}

www.sciencemag.org/cgi/content/full/336/6085/1182/DC1 Materials and Methods

Figs. S1 to S14

Table S1

References (29-32)

Movies S1 to S3

5 December 2011; accepted 24 April 2012 10.1126/science.1217416 